\title{
Effect of adalimumab on extraintestinal manifestations among patients with ulcerative colitis in a clinical practice setting: Results from INSPIRADA
}

\author{
Simon Travis, DPhil, FRCP ${ }^{1}$; Brian G. Feagan, $\mathrm{MD}^{2}$; Laurent Peyrin-Biroulet, MD, $\mathrm{PhD}^{3}$; Remo
} Panaccione, MD, FRCPC ${ }^{4}$, Silvio Danese, MD, PhD ${ }^{5}$; Andreas Lazar, MD ${ }^{6}$; Anne M. Robinson, PharmD ${ }^{7}$; Roopal B. Thakkar, $\mathrm{MD}^{7}$; Brandee Pappalardo, $\mathrm{PhD}^{7}$; Joel Petersson, PharmD, $\mathrm{PhD}^{7}$; Mareike Bereswill, $\mathrm{MSc}^{6}$; Naijun Chen, $\mathrm{MS}^{7}$; Martha Skup, $\mathrm{PhD}^{7}$

${ }^{1}$ Oxford University Hospitals, Oxford, United Kingdom

${ }^{2}$ Robarts Research Institute, Ontario, Canada

${ }^{3}$ University Hospital of Nancy, Les Nancy, France

${ }^{4}$ University of Calgary, Calgary, Canada

${ }^{5}$ Istituto Clinico Humanitas, Milan, Italy

${ }^{6}$ AbbVie Deutschland GmbH \& Co. KG, Ludwigshafen, Germany

${ }^{7}$ AbbVie Inc., North Chicago, IL, USA

Background: Extraintestinal manifestations (EIMs) are common in patients with ulcerative colitis (UC) and can have a significant impact on patient morbidity and quality of life ${ }^{1}$. We assessed the effect of adalimumab (ADA) therapy on the resolution of EIMs among patients with moderate to severe UC in clinical practice.

Methods: INSPIRADA was a single-arm, multi-country, open-label study evaluating the effect of ADA on clinical outcomes, costs of care, treatment satisfaction and work productivity in patients with UC treated according to usual clinical practice. Adults with active UC, Physician's Global Assessment (PGA) $\geq 2$ and Short Inflammatory Bowel Disease Questionnaire $\leq 45$ at baseline $(\mathrm{BL})$ who failed conventional treatment and who experienced rectal bleeding $\leq 7$ days of $B L$ were enrolled. Patients received 160/80 mg ADA at Week $0 / 2$ followed by $40 \mathrm{mg}$ of ADA every other week at Week 4 through Week 26. Patients who did not respond to ADA by Week 8 were to discontinue ADA. Patients who lost response at or after Week 8 could escalate to $40 \mathrm{mg}$ ADA weekly. The presence of EIMs (arthritis, pyoderma gangrenosum, erythema nodosum, and uveitis) were collected in the SCCAI at BL and Weeks 2, 8 and 26. McNemar's test was used to compare the presence of any EIM at Weeks 2, 8 and 26 with BL. In a subset of patients with EIMs at BL, EIM resolution was assessed at Week 2, 8 and 26, defined as no reported EIM at the respective visit; durable EIM resolution at Weeks 2 and 8 was defined as no EIM at the respective visit and subsequent visit(s). Missing data were imputed using last observation carried forward.

Results: Data from 461 patients were analysed. At BL, 88 patients (19.1\%) had an EIM. The most commonly reported EIM was arthritis (84 of 88 patients). Pyoderma gangrenosum, erythema nodosum and uveitis each were reported in $<1 \%$ of patients at BL and at Weeks 2,8 and 26 . The overall percentage of patients with any EIM decreased significantly $(P<.001)$ from BL over time: $13.2 \%, 11.7 \%$ and $10.8 \%$ had any EIMs at Weeks 2, 8 and 26, respectively. Similar decreasing percentages were seen for patients with arthritis: $13.0 \%, 11.7 \%$ and $10.8 \%$ at Weeks 2,8 and 26 , respectively. Among those with any EIM at BL, resolution of EIMs increased over time: $39.8 \%, 52.3 \%$ and $63.6 \%$ at Weeks 2, 8 and 26 , respectively; durable resolution was $23.9 \%$ and $44.3 \%$ at Weeks 2 and 8 , respectively. Among those with arthritis at BL, resolution rates were $36.9 \%, 50.0 \%$ and $61.9 \%$ at Weeks 2,8 and 26 , respectively; durable resolution was $20.2 \%, 41.7 \%$ and $61.9 \%$, respectively.

Conclusion: ADA therapy reduced EIMs among patients with moderate to severe UC in usual clinical practice, with EIM resolution in $60 \%$ by week 26 .

Reference: 1. Vavricka SR et al. Inflamm Bowel Dis 2015;21(8):1982-1992. 